\title{
ANÁLISE MULTIVARIADA DE ARGISSOLOS DA FAIXA ATLÂNTICA BRASILEIRA ${ }^{(1)}$
}

\author{
Waldir de Carvalho Junior ${ }^{(2)}$, Carlos Ernesto G.R. Schaefer ${ }^{(3)}$, César da Silva \\ Chagas $^{(2)} \&$ Elpídio Inácio Fernandes Filho ${ }^{(3)}$
}

\begin{abstract}
RESUMO
A faixa atlântica brasileira possui extrema diversidade ambiental, onde é expressiva a ocorrência de Argissolos, descritos e identifcados em diversos estudos, sem que tenham sido exauridas as possibilidades de interpretações. O objetivo deste estudo foi avaliar, por meio da análise multivariada, as principais diferenças nos atributos físicos e químicos de Argissolos da faixa atlântica brasileira. Foram analisados 91 perfis de solos oriundos de levantamentos pedológicos realizados pelo Projeto RADAMBRASIL, agrupados em três domínios geomorfológicos distintos: Planaltos Soerguidos, Planaltos Rebaixados e Tabuleiros Costeiros, por meio da análise de componentes principais e análise de função discriminante. Para isso, foram selecionadas 14 variáveis para identificar as peculiaridades de cada grupo geomorfológico e suas principais diferenças. A análise dos componentes principais demonstrou que os cinco primeiros componentes respondem por aproximadamente $70 \%$ da variação dos dados. A análise integrada confirmou que os Argissolos dos Planaltos Soerguidos são mais rasos, associando maiores taxas de erosão com menor espessura dos horizontes Bt, e são quimicamente pobres, embora mais rejuvenescidos e rasos. Já os Argissolos nos Planaltos Rebaixados, submetidos a um clima mais seco e, ou, sazonal que os situados nos Planaltos Soerguidos e Tabuleiros Costeiros, mostram-se menos intemperizados e com maior CTC. As análises realizadas (componentes principais e análise discriminante) permitem melhor entender as relações geomorfopedológicas dos Argissolos nos diferentes domínios estudados.
\end{abstract}

Termos para indexação: domínios geomorfológicos, componentes principais, análise discriminante, $B$ textural.

\footnotetext{
(1) Recebido para publicação em novembro de 2007 e aprovado em setembro de 2008.

(2) Pesquisador da Embrapa Solos. Rua Jardim Botânico 1024, Jardim Botânico, CEP 22460-000 Rio Janeiro (RJ). E-mails: waldircj@cnps.embrapa.br; chagas.rj@gmail.com

(3) Professor no Departamento de Solos, Universidade Federal de Viçosa - UFV. Av. P.H. Rolfs s/n, Campus Universitário, CEP 36570000 Viçosa (MG). E-mails: carlos.schaefer@ufv.br; elpidio@ufv.br
} 


\title{
SUMMARY: MULTIVARIATE ANALYSIS OF ARGISOLS OF THE BRAZILIAN ATLANTIC COAST
}

\begin{abstract}
The brazilian atlantic coast have has extreme environmental diversity, where is expressive the occurrence of Argisols, described and identified in several studies, that do not have exhausted the possibilities of interpretations. The objective of this study was to evaluate the main differences in the physical and chemical properties of Argisols of the Brazilian Atlantic coast by multivariate analysis, allowing inferences on their genesis and fertility. For this purpose, 91 soil profiles from the soil survey reports of the Project RADAMBRASIL were analyzed, and grouped into three distinct geomorphologic domains: Lower dissected plateaus - PR, High dissected plateaus - PS and Coastal tablelands - TC. These profiles were analyzed by principal components and discriminate functions. Fourteen variables were selected to identify peculiarities of each geomorphologic group and the main differences. The principal component analysis showed that the first five components account for approximately $70 \%$ of the data variation. The integrated analysis indicated that the Argisols of the high dissected plateaus are generally shallower and that the highest erosion rates are associated with the thinnest Bt horizons. But although younger and flatter, they are chemically poor. On the other hand, the Argisols in the lower dissected plateaus, in drier and/or more seasonal climates, are less weathered and have a higher CEC. The analyses (principal component and discriminate function analysis) allowed a better understanding of the pedogeomorphological relations of the Argisols in the different domains studied.
\end{abstract}

Index terms: geomorphologic domains, multivariate analysis, principal components.

\section{INTRODUÇÃO}

A Floresta Tropical Atlântica, também conhecida como Mata Atlântica, é encontrada ao longo da costa brasileira, desde o sul até o nordeste, avançando para o interior em extensões variadas. Seu passado de transgressões e retrações de domínios durante as oscilações climáticas do quaternário, associado aos diversos gradientes altitudinais, geomorfológicos, pedológicos, orométricos e latitudinais, conferiu ao panorama atual um quadro de extrema diversidade biológica, de hábitat e fitofisionomias florestais (Mantovani, 1993).

Nesse bioma, é expressiva a ocorrência de Argissolos (Embrapa, 2006) em diferentes condições geomorfológicas. Esta classe de solo foi descrita e identificada nos diversos levantamentos de solos realizados na região, principalmente pelo Projeto RADAMBRASIL. Apesar de sua importância e do considerável acervo de dados sobre esse solo, ainda hoje não foram exauridas todas as possibilidades de sua interpretação.

Nesse contexto, as análises de distribuição espacial, considerando as diferentes paisagens dos solos pelo uso da estatística multivariada, podem contribuir para a obtenção de melhor entendimento do ambiente (Boruvka \& Kozak, 2001). Dessa forma, a análise de componentes principais pode ser usada para identificar e quantificar a variação do solo associada a diferentes processos pedogenéticos.

A análise de componentes principais é um método de análise multivariada, utilizado para projetar dados n-dimensionais em um espaço de baixa dimensão. É um método exploratório que auxilia na elaboração de hipóteses gerais a partir dos dados coletados, contrastando com estudos direcionados nos quais hipóteses prévias são testadas. É também capaz de separar a informação importante daquela redundante e aleatória (Ferreira et al., 2002). Para Manly (1994), a análise de componentes principais é, resumidamente, um meio de simplificar os dados pela redução do número de variáveis.

A análise de componentes principais, muito utilizada para análise de dados de sensores remotos orbitais, tem sido também empregada para análise de atributos do solo. Splechtna \& Klinka (2001), estudando solos florestais em uma região montanhosa do Canadá, utilizaram a análise de componentes principais para examinar as relações entre regimes de nutrientes do solo identificados qualitativamente no campo e atributos de fertilidade do solo medidos em laboratório. Os resultados obtidos mostraram que os três primeiros componentes principais de todos os atributos de fertilidade estudados explicaram $60 \%$ da variância total entre todas as parcelas consideradas.

No Brasil, Couto \& Cunha (2002) utilizaram a análise fatorial dos componentes principais para integrar atributos químicos (15) e físicos (5) do solo para identificar pedopaisagens no Pantanal de Mato Grosso. Segundo esses autores, os primeiros quatro componentes explicaram cerca de 77 \% da variância total entre as pedopaisagens.

Gomes et al. (2004) utilizaram análise de componentes principais para avaliar diferenças de comportamento entre Latossolos e Neossolos Quartzarênicos relacionados com as superfícies 
geomórficas Sul-Americana e Velhas na região dos Cerrados, com o intuito de permitir a distinção mais detalhada das paisagens em condições naturais. Os autores concluíram que a análise realizada possibilitou melhor entendimento das diferenças e similaridades dos ambientes pedológicos separados no campo.

Assim, este estudo utilizou a análise de componentes principais e a análise discriminante na avaliação de atributos físicos e químicos de perfis de Argissolos, descritos nas Folhas do Projeto RADAMBRASIL que englobam uma parte da faixa atlântica entre os Estados da Bahia e São Paulo, buscando aprofundar o conhecimento sobre as relações pedogeomorfológicas desses solos, com o intuito de permitir melhor separação das diferentes pedopaisagens.

\section{MATERIAL E MÉTODOS}

A área estudada engloba uma parte da faixa atlântica brasileira compreendida entre a Bahia e São Paulo (Figura 1). Foram utilizados 91 perfis de Argissolos (53 perfis completos e 38 amostras extras) selecionados nas Folhas SD 24 - Salvador (Brasil, 1981); SE 24 - Rio Doce (Brasil, 1987) e SF 23/24 - Rio de Janeiro/Vitória (Brasil, 1983), relacionados a diferentes unidades geomorfológicas (Quadro 1).

As unidades geomorfológicas, definidas no conjunto de dados das folhas utilizadas, englobam uma ampla variação de formas e feições, como depressões, planaltos, tabuleiros e serras. Para atender aos objetivos do estudo, os perfis de solos utilizados foram agrupados de acordo com a unidade geomorfológica a que

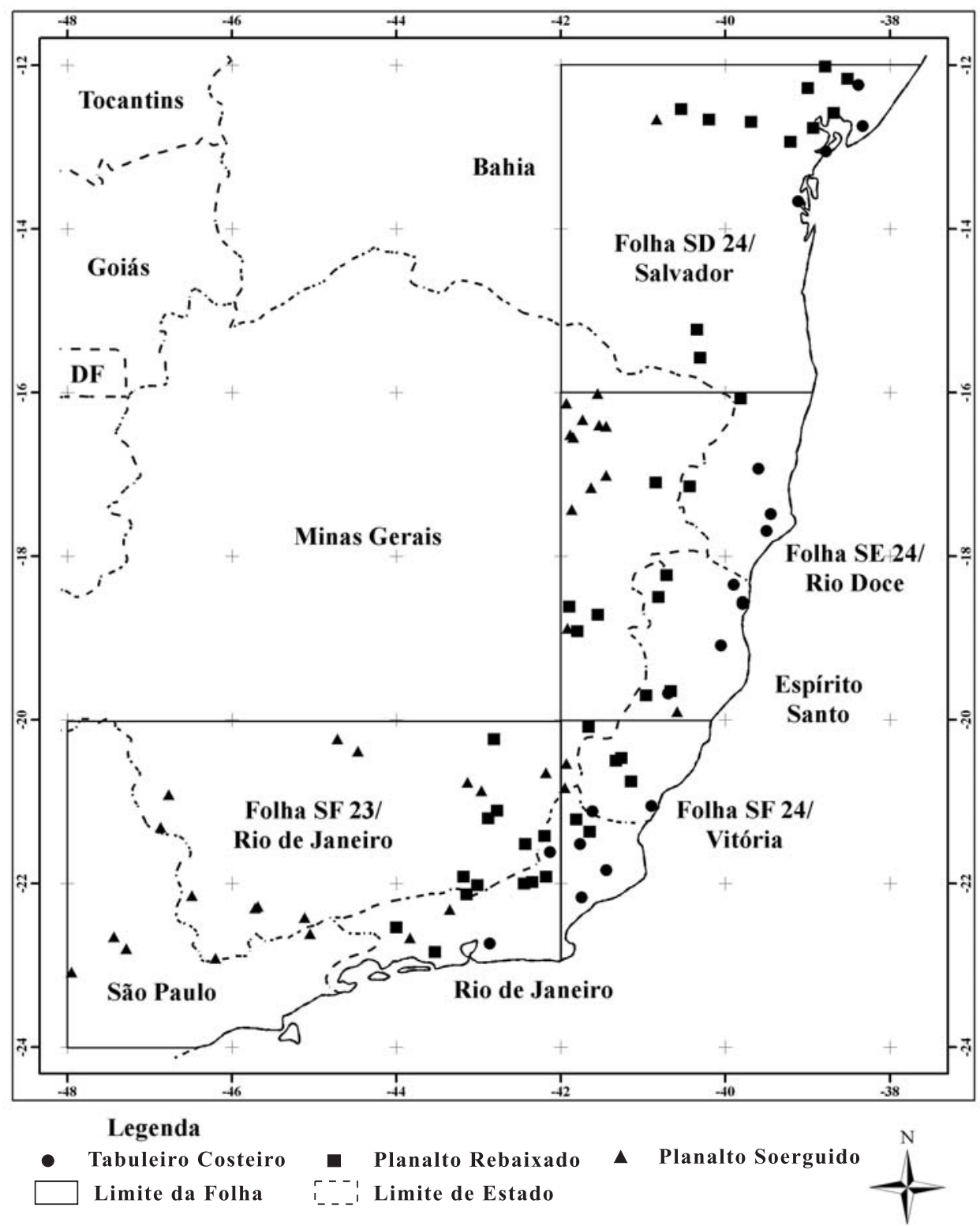

Figura 1. Localização dos perfis estudados nas Folhas do Projeto RADAMBRASIL. 
Quadro 1. Solos estudados em cada unidade geomorfológica

\begin{tabular}{|c|c|c|}
\hline Unidade geomorfológica & Classe de solo & Número de perfis utilizados \\
\hline Tabuleiros Costeiros & $\begin{array}{l}\text { Argissolo Amarelo } \\
\text { Argissolo Vermelho -Amarelo } \\
\text { Argissolo Vermelho }\end{array}$ & $\begin{array}{l}9 \\
8 \\
2\end{array}$ \\
\hline Planaltos Rebaixados & $\begin{array}{l}\text { Argissolo Acinzentado } \\
\text { Argissolo Vermelho -Amarelo } \\
\text { Argissolo Vermelho }\end{array}$ & $\begin{array}{r}1 \\
24 \\
11\end{array}$ \\
\hline Planaltos Soerguidos & $\begin{array}{l}\text { Argissolo Vermelho -Amarelo } \\
\text { Argissolo Vermelho }\end{array}$ & $\begin{array}{l}21 \\
15\end{array}$ \\
\hline
\end{tabular}

pertencem, conforme estabelecido nos levantamentos geomorfológicos do Projeto RADAMBRASIL. O enquadramento desses perfis foi realizado com o auxílio do mapa de solos e dos dados de elevação do Shuttle Radar Topographic Mission - SRTM (USGS, 2005).

As unidades consideradas foram: Domínio dos Planaltos Rebaixados (PR), Domínios dos Planaltos Soerguidos (PS) e Domínio dos Tabuleiros Costeiros (TC). De maneira geral, o Domínio dos Planaltos Rebaixados apresenta uma altitude máxima em torno de $400 \mathrm{~m}$. Os perfis localizados acima desta altitude foram considerados como pertencentes ao Domínio dos Planaltos Soerguidos. Já os perfis pertencentes ao Domínio dos Tabuleiros Costeiros encontram-se, normalmente, entre zero e $115 \mathrm{~m}$.

Após a formação dos grupos, cálculos estatísticos foram conduzidos sobre o conjunto de dados analíticos a fim de buscar correlações indicativas de características diferenciais entre os grupos e, mesmo, dentro dos grupos. Buscou-se estabelecer a associação entre distribuição espacial da classe dos Argissolos com as formas de relevo, independente da escala. Para tanto, os atributos físicos e químicos considerados nesta análise (horizonte Bt) foram: espessura do horizonte, areia grossa, areia fina, silte, argila, gradiente textural, argila dispersa em água, relação silte/argila, $\mathrm{pH}$ em $\mathrm{H}_{2} \mathrm{O}, \mathrm{pH}$ em $\mathrm{KCl}, \Delta \mathrm{pH}, \mathrm{Ca}^{2+}, \mathrm{Mg}^{2+}$, $\mathrm{K}^{+}, \mathrm{Na}^{+}$, soma de bases, $\mathrm{Al}^{3+}, \mathrm{H}^{+}, \mathrm{H}+\mathrm{Al}$, CTC efetiva, valor $\mathrm{T}$, atividade da fração argila, valor $\mathrm{V}, \mathrm{C}$ orgânico, $\mathrm{N}$, relação $\mathrm{C} / \mathrm{N}, \mathrm{SiO}_{2}, \mathrm{Al}_{2} \mathrm{O}_{3}, \mathrm{Fe}_{2} \mathrm{O}_{3}, \mathrm{TiO}_{2}, \mathrm{Ki}, \mathrm{Kr}$ e relação $\mathrm{Al}_{2} \mathrm{O}_{3} / \mathrm{Fe}_{2} \mathrm{O}_{3}$.

Visto que as variáveis de interesse se comportam de maneira independente, foi realizada análise de componentes principais para reduzir o número delas. Posteriormente, a análise de função discriminante, técnica estatística que permite estudar diferenças entre dois ou mais grupos, em função de um conjunto de informações conhecidas para todos os elementos dos grupos, foi utilizada para mostrar as variações estatísticas entre os grupos de forma geral. Todos os cálculos estatísticos foram feitos utilizando-se o sistema Statistica versão 6.0.
A análise discriminante trata de uma técnica estatística multivariada usada na resolução de problemas que envolvem a separação de conjuntos distintos de objetos, ou observações, e a alocação de novos objetos, ou observações em um grupo específico.

A redução do número de variáveis a serem analisadas foi feita por meio da avaliação da matriz de correlação, que é uma medida da relação entre duas ou mais variáveis, e da análise de componentes principais, considerando todos os casos, independente da unidade geomorfológica a que pertencem e da importância deles no Sistema Brasileiro de Classificação de Solos (Embrapa, 2006) para tal redução.

\section{RESULTADOS E DISCUSSÃO}

Analisando o grupo das variáveis associadas à textura, a variável silte ( \%) apresentou coeficientes de correlação significativo com a relação silte/argila (Quadro 2). Assim, as variáveis argila e silte foram mantidas. A variável $\Delta \mathrm{pH}$ mostrou coeficiente de correlacão significativo com a CTC efetiva, soma de bases, valor T e índices Ki e Kr (Quadro 3). Dessa forma, os valores de $\mathrm{pH}$ em água e em $\mathrm{KCl}$ foram descartados, utilizando-se apenas o $\Delta \mathrm{pH}$.

Como os teores de $\mathrm{Ca}^{2+}, \mathrm{Mg}^{2+}, \mathrm{K}^{+}$e $\mathrm{Na}^{+}$mostram coeficiente de correlação significativo com a soma de bases, utilizou-se apenas esta última. Já o teor de $\mathrm{Al}^{3+}$, que representa um caráter de classificação em níveis hierárquicos mais baixos, mostrou valores de correlação significativos com $\mathrm{H}^{+} \mathrm{e} \mathrm{H}+\mathrm{Al}$, e mantendose somente o $\mathrm{Al}^{3+}$ (Quadro 3). A CTC efetiva mostrou coeficiente de correlação significativo tanto com a soma de bases quanto com o valor T. Nesse caso, foram mantidos o valor T e a CTC efetiva, pois trata-se de atributos utilizados na classificação e para possíveis interpretações. Com relação ao ataque sulfúrico, os índices $\mathrm{Ki}$ e $\mathrm{Kr}$ e os teores de $\mathrm{Fe}_{2} \mathrm{O}_{3}$ e $\mathrm{TiO}_{2}$ foram utilizados na análise estatística, para auxiliar na interpretação final dos grupos de dados. 
Quadro 2. Estatística descritiva indicando o número de amostras válidas, valores de média máximo e mínimo, desvio-padrão e curtose para as variáveis do horizonte Bt

\begin{tabular}{|c|c|c|c|c|c|c|}
\hline Variável & $\mathrm{N}^{\circ}$ de amostras & Média & Mínimo & Máximo & Desvio-padrão & Curtose \\
\hline Espessura do horizonte $\mathrm{Bt}(\mathrm{cm})$ & 91 & 62,11 & 10,00 & 250,00 & 49,90 & 3,28 \\
\hline Argila (\%) & 91 & 51,61 & 16,00 & 76,00 & 14,32 & $-0,38$ \\
\hline Silte $(\%)$ & 91 & 11,60 & 1,00 & 32,00 & 6,20 & 0,75 \\
\hline Argila natural (\%) & 90 & 11,94 & 0,00 & 60,00 & 15,96 & 1,18 \\
\hline$\Delta \mathrm{pH}$ & 91 & $-0,86$ & $-1,80$ & $-0,08$ & 0,36 & 0,29 \\
\hline CTC efetiva $\left(\mathrm{cmol}_{\mathrm{c}} \mathrm{dm}^{-3}\right)$ & 91 & 3,50 & 0,33 & 33,05 & 4,08 & 30,59 \\
\hline Soma de Bases & 91 & 2,46 & 0,13 & 16,60 & 2,82 & 8,84 \\
\hline $\mathrm{Al}^{3+}\left(\mathrm{cmol}_{\mathrm{c}} \mathrm{dm}^{-3}\right)$ & 91 & 1,04 & 0,00 & 16,45 & 2,28 & 25,60 \\
\hline Valor $\mathrm{T}\left(\mathrm{cmol}_{\mathrm{c}} \mathrm{dm}^{-3}\right)$ & 91 & 5,29 & 0,00 & 35,85 & 4,52 & 23,22 \\
\hline Valor V (\%) & 90 & 40,12 & 3,00 & 100,00 & 25,79 & $-0,98$ \\
\hline C orgânico (\%) & 91 & 0,40 & 0,11 & 1,05 & 0,18 & 1,66 \\
\hline $\mathrm{N}\left(\mathrm{g} \mathrm{kg}^{-1}\right)$ & 87 & 0,07 & 0,00 & 0,47 & 0,05 & 40,61 \\
\hline $\mathrm{Al}_{2} \mathrm{O}_{3}(\mathrm{~g} \mathrm{~kg}-1)$ & 88 & 18,89 & 5,70 & 29,48 & 5,24 & $-0,34$ \\
\hline $\mathrm{Fe}_{2} \mathrm{O}_{3}\left(\mathrm{~g} \mathrm{~kg}^{-1}\right)$ & 88 & 6,40 & 0,77 & 16,30 & 3,46 & $-0,61$ \\
\hline $\mathrm{TiO}_{2}\left(\mathrm{~g} \mathrm{~kg}^{-1}\right)$ & 83 & 0,91 & 0,00 & 2,18 & 0,51 & $-0,25$ \\
\hline $\mathrm{Ki}$ & 88 & 2,02 & 0,49 & 3,27 & 0,39 & 3,34 \\
\hline $\mathrm{Kr}$ & 88 & 1,66 & 0,41 & 2,65 & 0,32 & 2,88 \\
\hline Gradiente textural & 91 & 2,45 & 1,14 & 8,86 & 1,43 & 7,09 \\
\hline
\end{tabular}

Quadro 3. Matriz de correlação com valores significativos $(p<0,05)$ realçados, para as variáveis selecionadas do horizonte $\mathrm{Bt}$

\begin{tabular}{|c|c|c|c|c|c|c|c|c|c|c|c|c|c|c|c|c|c|c|}
\hline Variável & Esp & Sil & Arg & $\mathrm{B} / \mathrm{A}$ & Arg Nat. &.$\Delta \mathrm{pH}$ & SB & $\mathrm{Al}^{3+}$ & CTCe & $\mathbf{T}$ & $\mathrm{V}$ & C & $\mathrm{N}$ & $\mathrm{Al}_{2} \mathrm{O}_{3}$ & $\mathrm{Fe}_{2} \mathrm{O}_{3}$ & $\mathrm{TiO}_{2}$ & $\mathbf{K i}$ & $\mathbf{K r}$ \\
\hline Esp & 1,00 & & & & & & & & & & & & & & & & & \\
\hline Sil & $-0,16$ & 1,00 & & & & & & & & & & & & & & & & \\
\hline Arg & $-0,22$ & 0,04 & 1,00 & & & & & & & & & & & & & & & \\
\hline $\mathrm{B} / \mathrm{A}$ & 0,12 & $-0,37$ & $-0,01$ & 1,00 & & & & & & & & & & & & & & \\
\hline Arg Nat. & $-0,07$ & 0,03 & 0,10 & 0,05 & 1,00 & & & & & & & & & & & & & \\
\hline$\Delta \mathrm{pH}$ & 0,13 & $-0,13$ & 0,18 & 0,10 & $-0,44$ & 1,00 & & & & & & & & & & & & \\
\hline $\mathrm{SB}$ & $-0,06$ & 0,41 & 0,22 & $-0,09$ & 0,42 & $-0,52$ & 1,00 & & & & & & & & & & & \\
\hline $\mathrm{Al}$ & 0,08 & 0,26 & 0,08 & 0,05 & 0,20 & $-0,34$ & 0,20 & 1,00 & & & & & & & & & & \\
\hline CTCe & $-0,09$ & 0,42 & 0,19 & $-0,09$ & 0,43 & $-0,57$ & 0,87 & 0,76 & 1,00 & & & & & & & & & \\
\hline $\mathrm{T}$ & $-0,17$ & 0,41 & 0,25 & $-0,09$ & 0,41 & $-0,52$ & 0,80 & 0,78 & 0,95 & 1,00 & & & & & & & & \\
\hline $\mathrm{V}$ & 0,05 & 0,29 & 0,18 & $-0,07$ & 0,26 & $-0,16$ & 0,64 & $-0,24$ & 0,36 & 0,23 & 1,00 & & & & & & & \\
\hline $\mathrm{CO}(\%)$ & $-0,26$ & 0,34 & 0,21 & $-0,27$ & 0,31 & $-0,07$ & 0,12 & 0,27 & 0,19 & 0,22 & 0,04 & 1,00 & & & & & & \\
\hline $\mathrm{N}$ & $-0,03$ & 0,18 & 0,09 & $-0,28$ & 0,20 & $-0,24$ & 0,25 & 0,14 & 0,26 & 0,22 & 0,27 & 0,46 & 1,00 & & & & & \\
\hline $\mathrm{Al}_{2} \mathrm{O}_{3}$ & $-0,09$ & 0,19 & 0,72 & $-0,09$ & $-0,08$ & 0,11 & $-0,05$ & $-0,04$ & $-0,06$ & $-0,04$ & $-0,06$ & 0,09 & $-0,00$ & 1,00 & & & & \\
\hline $\mathrm{Fe}_{2} \mathrm{O}_{3}$ & $-0,03$ & 0,28 & 0,61 & $-0,28$ & $-0,09$ & 0,19 & 0,34 & $-0,04$ & 0,24 & 0,29 & 0,36 & 0,24 & 0,16 & 0,20 & 1,00 & & & \\
\hline $\mathrm{TiO}_{2}$ & $-0,03$ & $-0,08$ & 0,43 & 0,02 & $-0,03$ & $-0,08$ & 0,00 & $-0,05$ & $-0,02$ & $-0,06$ & 0,04 & $-0,04$ & $-0,03$ & $-0,00$ & $-0,01$ & 1,00 & & \\
\hline $\mathrm{Ki}$ & $-0,05$ & 0,18 & 0,07 & 0,01 & 0,32 & $-0,54$ & 0,58 & 0,46 & 0,61 & 0,52 & 0,41 & $-0,13$ & 0,11 & $-0,09$ & $-0,06$ & 0,11 & 1,00 & \\
\hline $\mathrm{Kr}$ & 0,00 & 0,06 & $-0,07$ & 0,17 & 0,34 & $-0,47$ & 0,30 & 0,41 & 0,37 & 0,27 & 0,21 & $-0,22$ & 0,01 & $-0,15$ & $-0,40$ & 0,10 & 0,85 & 1,00 \\
\hline
\end{tabular}

Esp: espessura; Sil: silte; Arg: argila; B/A: relação textural; Arg Nat.: argila natural; $\Delta \mathrm{pH}$ : $\mathrm{pH} \mathrm{H}_{2} \mathrm{O}-\mathrm{pH}$ KCl; SB: soma de bases; $\mathrm{Al}^{3+}$ : alumínio trocável; CTCe: CTC efetiva; T: Valor T; V: Valor V; CO: carbono orgânico; e N: nitrogênio. 
Dessa forma, após a análise da matriz de correlação entre as variáveis com seus valores padronizados e da ponderação de sua importância no Sistema Brasileiro de Classificação de Solos (Embrapa, 2006), reduziu-se aproximadamente 50 \% do número de variáveis, passando de 34 variáveis iniciais para 18. Para esse grupo de variáveis, foi então realizada a análise de componentes principais (ACP), que determinou que os cinco primeiros componentes principais respondem por aproximadamente $70 \%$ da variação dos dados, sendo $28,29 \%$ para o fator 1 ; $18,28 \%$ para o fator $2 ; 10,08 \%$ para o fator $3 ; 8 \%$ para o fator 4 e $7,07 \%$ para o fator 5 (Quadro 4). Entretanto, na definição das variáveis foi utilizado até o terceiro fator.

Assim, analisando a primeira componente principal (fator 1) em termos de contribuição das variáveis, pode-se verificar que as variáveis CTC efetiva, T, SB, $\mathrm{Ki}, \Delta \mathrm{pH}$ e $\mathrm{Kr}$ são, nesta ordem, as que mais contribuem para a definição desse fator, somando em conjunto 0,74 do total de uma unidade (Quadro 5). Para a segunda componente principal, verificou-se que as variáveis que mais contribuem são $\mathrm{Fe}_{2} \mathrm{O}_{3}, \mathrm{Al}_{2} \mathrm{O}_{3}$, argila e $\mathrm{TiO}_{2}$, somando aproximadamente 0,71 de peso neste fator. Para o fator 3 , ou terceira componente principal, observou-se que as variáveis $\mathrm{C}$ orgânico,

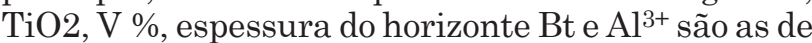
maior peso.

Com base nesses resultados, selecionaram-se 14 variáveis (CTC efetiva, T, soma de bases, $\mathrm{Ki}, \Delta \mathrm{pH}$, $\mathrm{Kr}, \mathrm{Fe}_{2} \mathrm{O}_{3}, \mathrm{Al}_{2} \mathrm{O}_{3}$, argila, $\mathrm{TiO}_{2}, \mathrm{C}$ orgânico, $\mathrm{V} \%$, espessura $\mathrm{Al}^{3+}$ ) para identificar as peculiaridades de cada Domínio geomorfológico, bem como as principais diferenças. A análise estatística descritiva para o conjunto de dados de cada Domínio geomorfológico indica diferenças entre os grupos, principalmente em relação à soma de bases, valor $\mathrm{T}, \mathrm{Ki}, \mathrm{Kr}, \mathrm{Al}_{2} \mathrm{O}_{3}, \mathrm{C}$ orgânico, CTC efetiva, espessura e relação textural.

Quadro 4. Autovalores dos fatores (componentes principais) e das estatísticas correlatas

\begin{tabular}{|c|c|c|c|c|}
\hline Fator & Autovalor & $\begin{array}{c}\% \\
\text { Total }\end{array}$ & $\begin{array}{c}\text { Autovalor } \\
\text { Cumulativo }\end{array}$ & $\begin{array}{c}\% \\
\text { Cumulativa }\end{array}$ \\
\hline 1 & 5,09 & 28,29 & 5,09 & 28,29 \\
\hline 2 & 3,29 & 18,28 & 8,38 & 46,57 \\
\hline 3 & 1,81 & 10,08 & 10,20 & 56,65 \\
\hline 4 & 1,44 & 8,00 & 11,64 & 64,65 \\
\hline 5 & 1,27 & 7,07 & 12,91 & 71,72 \\
\hline 6 & 1,09 & 6,06 & 14,00 & 77,78 \\
\hline 7 & 0,92 & 5,11 & 14,92 & 82,89 \\
\hline 8 & 0,76 & 4,20 & 15,68 & 87,09 \\
\hline 9 & 0,65 & 3,63 & 16,33 & 90,72 \\
\hline 10 & 0,50 & 2,75 & 16,82 & 93,47 \\
\hline 11 & 0,39 & 2,17 & 17,22 & 95,64 \\
\hline 12 & 0,29 & 1,63 & 17,51 & 97,28 \\
\hline 13 & 0,20 & 1,11 & 17,71 & 98,39 \\
\hline 14 & 0,15 & 0,82 & 17,86 & 99,21 \\
\hline 15 & 0,07 & 0,38 & 17,93 & 99,59 \\
\hline 16 & 0,04 & 0,22 & 17,97 & 99,81 \\
\hline 17 & 0,03 & 0,19 & 18,00 & 100,00 \\
\hline 18 & 0,00 & 0,00 & 18,00 & 100,00 \\
\hline
\end{tabular}

Quadro 5. Contribuição de cada variável, baseada na covariância, para cada um dos cinco primeiros componentes principais (fator)

\begin{tabular}{|c|c|c|c|c|c|}
\hline Variável & Fator 1 & Fator 2 & Fator 3 & Fator 4 & Fator 5 \\
\hline Espessura do horizonte Bt & 0,0076 & 0,0091 & 0,0747 & 0,0002 & 0,0000 \\
\hline Argila & 0,0019 & 0,1764 & 0,0011 & 0,0109 & 0,1815 \\
\hline Silte & 0,0424 & 0,0226 & 0,0333 & 0,0279 & 0,1711 \\
\hline Argila natural & 0,0587 & 0,0044 & 0,0295 & 0,0330 & 0,1047 \\
\hline$\Delta \mathrm{pH}$ & 0,0982 & 0,0353 & 0,0054 & 0,0009 & 0,0001 \\
\hline CTC efetiva & 0,1718 & 0,0016 & 0,0017 & 0,0408 & 0,0015 \\
\hline Soma de Bases & 0,1448 & 0,0072 & 0,0499 & 0,0089 & 0,0033 \\
\hline $\mathrm{Al}^{3+}$ & 0,0589 & 0,0026 & 0,0685 & 0,3001 & 0,0001 \\
\hline $\mathrm{T}$ & 0,1524 & 0,0070 & 0,0000 & 0,0902 & 0,0014 \\
\hline $\mathrm{V}(\%)$ & 0,0418 & 0,0172 & 0,1169 & 0,2685 & 0,0006 \\
\hline $\mathrm{CO}$ & 0,0108 & 0,0426 & 0,2699 & 0,0192 & 0,0065 \\
\hline $\mathrm{N}$ & 0,0195 & 0,0098 & 0,0637 & 0,0840 & 0,0007 \\
\hline $\mathrm{Al}_{2} \mathrm{O}_{3}$ & 0,0034 & 0,1807 & 0,0291 & 0,0137 & 0,0905 \\
\hline $\mathrm{Fe}_{2} \mathrm{O}_{3}$ & 0,0056 & 0,2375 & 0,0402 & 0,0035 & 0,0097 \\
\hline $\mathrm{TiO}_{2}$ & 0,0004 & 0,1126 & 0,1394 & 0,0663 & 0,0032 \\
\hline $\mathrm{Ki}$ & 0,1121 & 0,0268 & 0,0527 & 0,0039 & 0,0066 \\
\hline $\mathrm{Kr}$ & 0,0655 & 0,0836 & 0,0072 & 0,0143 & 0,0457 \\
\hline Gradiente textural & 0,0042 & 0,0228 & 0,0171 & 0,0138 & 0,3729 \\
\hline
\end{tabular}


De acordo com os resultados apresentados na matriz de classificação da análise discriminante, estatisticamente os agrupamentos estão corretos em pelo menos 66,7 \% (grupo dos Tabuleiros Costeiros), chegando a $82,9 \%$ para o grupo dos Planaltos Rebaixados (Quadro 6). A análise dos resultados estatísticos permite inferir tendências de comportamento entre os grupos de Argissolos dos Domínios geomorfológicos estudados.

Lima et al. (2004), estudando Argissolos dos Tabuleiros Costeiros, constataram a baixa disponibilidade de nutrientes associada à alta saturação por $\mathrm{Al}^{3+}$ e alta acidez ativa e trocável. Tal fato é corroborado nos dados analisados, por meio dos valores de soma de bases, com 1,2 de média contra 3,9 para o Planalto Rebaixado e 2,0 para o Planalto Soerguido. Os valores de $\mathrm{Al}^{3+}$ dos Argissolos estudados indicam, em termos de média, que aqueles pertencentes aos Domínios dos Planaltos Rebaixados e Tabuleiros Costeiros tendem a ser mais elevados (respectivamente, 1,08 e 1,04) do que os dos Planaltos Soerguidos $(0,87)$. Eles observaram também que os Argissolos dos Tabuleiros Costeiros possuem saturação por bases baixa $(\mathrm{V}<50 \%$ ) e o Ki igual ou maior que 1,7 , fato constatado nos dados analisados, com médias de V \% de $29 \%$ e Ki igual a 1,98.

Os Argissolos dos Tabuleiros Costeiros mostramse mais profundos do que os dos Planaltos Soerguidos e Planaltos Rebaixados, evidenciando a deposição de sedimentos pré-intemperizados oriundos dos Domínios geomorfológicos mais elevados e transportados, e sedimentados na faixa costeira, onde os sedimentos continuam sob a ação da pedogênese, mas com menor taxa de erosão condicionada pelo relevo baixo e tabular. Os dados evidenciam a maior espessura do horizonte Bt dos Tabuleiros Costeiros tendo média de $85 \mathrm{~cm}$, contra 63 e $43 \mathrm{~cm}$ dos Planaltos Rebaixados e Planaltos Soerguidos, respectivamente, mostrando a tendência de maior desenvolvimento pedogenético em profundidade nos compartimentos de planaltos mais baixos.

No Domínio dos Planaltos Soerguidos, de paisagem erosional intensamente dissecada, caracterizada pela correlação estreita entre a estrutura das rochas e as feições do relevo (Modenesi-Gauttieri \& Toledo, 1996), as maiores taxas de erosão nos Argissolos estão associadas à menor espessura dos horizontes Bt (Figura 2). Neste ambiente, também se espera maior taxa de produção e transporte de sedimentos, onde os processos pedogenéticos não conseguem superar a taxa dos processos morfogenéticos.

Nos Planaltos Soerguidos, rochas alcalinas podem facilmente ser intemperizadas para materiais cauliníticos e gibsíticos, com quartzo residual, resultando em baixa relação sílica/alumina da fração argila (Modenesi-Gauttieri et al., 2002). Assim, o Ki médio para os Planaltos Soerguidos foi de 1,96 contra 2,14 para os dados relacionados aos Planaltos Rebaixados. O Ki parece refletir o ambiente mais seco sazonal dos Planaltos Rebaixados, em relação aos compartimentos mais úmidos e elevados dos Planaltos Soerguidos, ou mais úmidos e tabulares dos Tabuleiros Costeiros.

Birkeland et al. (2003), em estudo realizado no Colorado (EUA), observaram que a distribuição altitudinal dos solos mostra o impacto dos fatores clima e vegetação. Segundo os dados analisados, a principal evidência de que os processos pedogenéticos ocorrem numa taxa inferior aos processos morfogenéticos nos Planaltos Rebaixados em relação aos Tabuleiros Costeiros é a comparação entre a espessura média do horizonte Bt (Figura 2).

O relevo do Sudeste brasileiro mostra claramente a herança dos processos tectônicos do período Cenozóico, que se seguiram à reativação MesozóicoCenozóica e a abertura do oceano Atlântico Sul (Saadi, 1993; Schaefer et al., 2002; Modenesi-Gauttieri et al., 2002). Corroborando com Schaefer et al. (2000), este trabalho demonstra a proeminência dos fatores morfoclimáticos sobre blocos mais soerguidos, guiando a evolução geomórfica dos Planaltos Soerguidos, que constrastam com aqueles processos que ocorreram nos Planaltos Rebaixados; já os Tabuleiros Costeiros são geoformas bem mais tardias, relacionadas com ciclos de deposição associados às oscilações climáticas do Quaternário.

Inseridos num sistema considerado mais dinâmico, os Argissolos do Domínio dos Planaltos Soerguidos apresentam atributos correlacionados aos processos geomórficos atuantes que conduziram a menor

Quadro 6. Matriz de classificação, com as linhas representando a classificação estatística observada e as colunas representado a classificação geomorfológica predita

\begin{tabular}{lccccc}
\hline $\begin{array}{c}\text { Domínio } \\
\text { geomorfológico }\end{array}$ & $\%$ & $\begin{array}{c}\text { Tabuleiros } \\
\text { Costeiros }\end{array}$ & $\begin{array}{c}\text { Planaltos } \\
\text { Rebaixados }\end{array}$ & $\begin{array}{c}\text { Planaltos } \\
\text { Soerguidos }\end{array}$ & $\begin{array}{c}\text { Total } \\
\text { Estatístico }\end{array}$ \\
\hline Tabuleiros Costeiros & 66,7 & 12 & 6 & 0 & 1 \\
Planaltos Rebaixados & 82,9 & 5 & 29 & 7 & 21 \\
Planaltos Soerguidos & 72,4 & 1 & 42 & 22 \\
Total predito & 75,6 & 18 & & & 29 \\
\hline
\end{tabular}



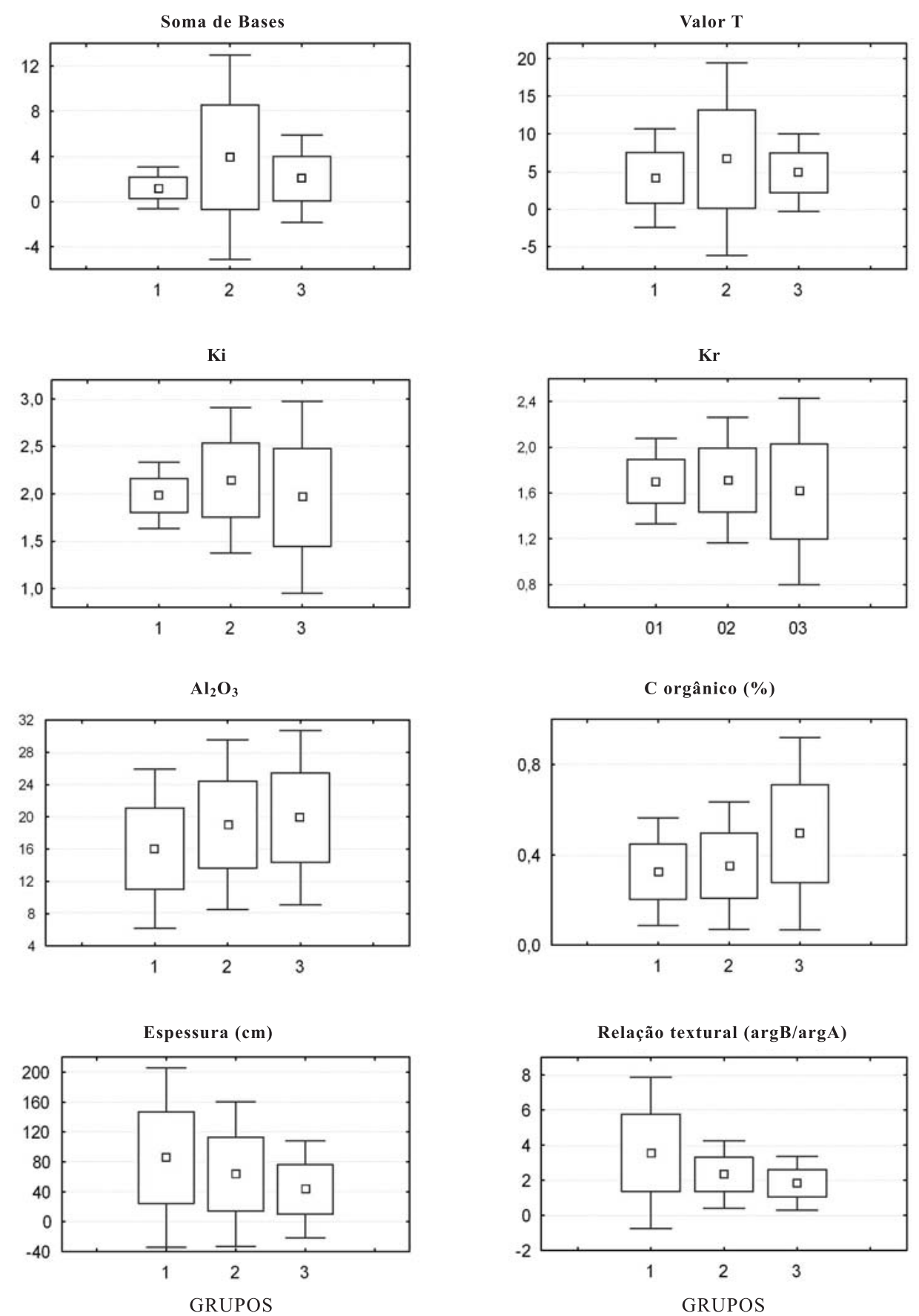

Figura 2. Representação gráfica da média, desvio-padrão e intervalo de confiança (desvio-padrão x 1,96) das variáveis entre os grupos definidos: 1 = TC, 2 = PR, e $3=$ PS. Onde: $\square$ média; $\square \pm$ desvio-padrão e I intervalo de confiança

espessura do sólum e maior taxa de rejuvenescimento pelas condições de relevo forte ondulado e montanhoso e pela altitude condicionando peculiaridades climáticas. Os Argissolos, além de mais rasos, possuem melhor fertilidade natural que aqueles desenvolvidos a partir dos sedimentos dos Tabuleiros Costeiros, pois o intemperismo atua sobre o saprolito ou diretamente sobre o material de origem, que está mais próximo à superfície.
Considerando o C orgânico, observa-se tendência de aumento no valor dessa variável dos Tabuleiros Costeiros em relação à dos Planaltos Soerguidos, em função do fator clima, com temperatura e precipitação pluvial marcadamente diferentes, provocando diferentes aportes e graus de ciclagem da matéria orgânica no solo. Verifica-se o aumento de $0,32 \%$ de C orgânico nos Tabuleiros Costeiros para 0,49 \%, nos teores médios, nos Planaltos Soerguidos. 
$\mathrm{O}$ teor médio de $\mathrm{Al}_{2} \mathrm{O}_{3}$ também apresenta tendência de aumento no sentido dos Tabuleiros Costeiros para os Planaltos Rebaixados e Planaltos Soerguidos. A relação textural mostra tendência de ser menor nos Planaltos Soerguidos e ir crescendo em direção aos Tabuleiros Costeiros. O gradiente textural médio tende a diminuir no sentido dos Tabuleiros Costeiros para os Planaltos Rebaixados e Planaltos Soerguidos. Assim, os Argissolos dos Tabuleiros Costeiros apresentaram valor médio de 3,6, enquanto o dos Planaltos Soerguidos foi de 1,8.

Pode-se dizer que a gênese do gradiente textural nesses solos está associada à intensa morfogênese (erosão) nos Planaltos Soerguidos e Planaltos Rebaixados, enquanto nos Argissolos dos Tabuleiros Costeiros parece ocorrer processos pedogenéticos em relevo plano, com menores taxas de erosão, como a translocação de argila e a ferrólise.

\section{CONCLUSÕES}

1. A estatística multivariada utilizada, por meio de análise de componentes principais e análise discriminante, possibilitou melhor entendimento das relações geomorfopedológicas dos Argissolos dos diferentes domínios geomorfológicos estudados.

2. A análise de componentes principais evidenciou que os cinco primeiros componentes principais respondem por aproximadamente $70 \%$ da variação dos dados. Dentre os três primeiros as variáveis mais importantes são CTC efetiva, valor T, soma de bases, $\mathrm{Ki}, \Delta \mathrm{pH}, \mathrm{Kr}, \mathrm{Fe}_{2} \mathrm{O}_{3}, \mathrm{Al}_{2} \mathrm{O}_{3}$, argila, $\mathrm{TiO}_{2}, \mathrm{C}$ orgânico, $\mathrm{V}$, espessura e $\mathrm{Al}^{3+}$.

3. Os resultados mostraram que os Argissolos dos Planaltos Soerguidos são mais rasos e de maior fertilidade natural que os Argissolos dos Tabuleiros Costeiros.

4. Os Argissolos nos Planaltos Rebaixados, submetidos a um clima mais seco e, ou, sazonais que os situados nos Planaltos Soerguidos e Tabuleiros Costeiros, mostram-se menos intemperizados e com maior CTC.

5. Os teores de $\mathrm{C}$ orgânico e $\mathrm{Al}_{2} \mathrm{O}_{3}$ aumentam no sentido dos Tabuleiros Costeiros para os Planaltos Rebaixados e Planaltos Soerguidos.

\section{LITERATURA CITADA}

BIRKELAND, P.W.; SHROBAB, R.R.; BURNSC, S.F.; PRICED A.B. \& TONKINE, P.J. Integrating soils and geomorphology in mountains - An example from the Front Range of Colorado. Geomorphology, 55:329-344, 2003.

BORUVKA, L. \& KOZAK, J. Geostatistical investigation of a reclaimed dumpsite soil with emphasis on aluminum. Soil Till. Res., 59:115-126, 2001.
BRASIL. Ministério de Minas e Energia. Secretaria-Geral. Projeto RADAMBRASIL. Folha SD. 24 Salvador; geologia, geomorfologia, pedologia, vegetação e uso potencial da terra. Rio de Janeiro, 1981. 624p.

BRASIL. Ministério de Minas e Energia. Secretaria-Geral. Projeto RADAMBRASIL. Folha SF. 23/24 Rio de Janeiro/ Vitória; geologia, geomorfologia, pedologia, vegetação e uso potencial da terra. Rio de Janeiro, 1983. 780p.

BRASIL. Ministério de Minas e Energia. Secretaria-Geral. Projeto RADAMBRASIL. Folha SE. 24 Rio Doce; geologia, geomorfologia, pedologia, vegetação e uso potencial da terra. Rio de Janeiro, 1987. 544p.

COUTO, E.G. \& CUNHA, C.N. Application of multivariate analysis to identify soil landscapes in the Pantanal of Mato Grosso - Brazil. In: WORLD CONGRESS OF SOIL SCIENCE, 17., Bangkok, 2002. Proceedings. Bangkok, 2002. v.1. p.1-10

EMPRESA BRASILEIRA DE PESQUISA AGROPECUÁRIA EMBRAPA. Centro Nacional de Pesquisa de Solos. Sistema brasileiro de classificação de solos. Rio de Janeiro, 2006. 306p.

FERREIRA, E.C.; RODRIGUES, S.H.B.G.; FERREIRA, M.M.C.; NÓBREGA, J.A. \& NOGUEIRA, A.R.A. Análise exploratória dos teores de constituintes inorgânicos em sucos e refrigerantes de uva. Eclética Química, 27:77-90, 2002 .

GOMES, J.B.V.; CURI, N.; MOTTA, P.E.F.; KER, J.C.; MARQUES, J.J.G.S.M. \& SCHULZE, D.G. Análise de componentes principais de atributos físicos, químicos e mineralógicos de solos do bioma Cerrado. R. Bras. Ci. Solo, 28:137-153, 2004.

LIMA, H.V.; SILVA, A.P.; JACOMINE, P.T.K.; ROMERO, R.E \& LIBARDI, P.L. Identificação e caracterização de solos coesos no Estado do Ceará. R. Bras. Ci. Solo, 28:467-476, 2004 .

MANLY, B.F.J. Multivariate statistical methods: A primer. 2.ed. New York, Chapman \& Hall, 1994. 215p.

MANTOVANI, W. Estrutura e dinâmica da floresta atlântica na Juréia, Iguape - SP. São Paulo, Universidade de São Paulo, 1993. 126p. (Tese de Livre-Docência)

MODENESI-GAUTTIERI, M.C.; HIRUMA, S.T. \& RICCOMINI, C. Morphotectonics of a high plateau on the Northwestern flank of the Continental Rift of Southeastern Brazil. Geomorphology, 43:257-271, 2002.

MODENESI-GAUTTIERI, M.C. \& TOLEDO, M.C.M. Weathering and the formation of hillslopes deposits in the tropical highlands of Itatiaia - Southeastern Brazil. Catena, 27:81-103, 1996.

SAADI, A. Neotectônica da plataforma brasileira: Esboço e interpretação preliminares. Geonomos, 1:1-15, 1993.

SCHAEFER, C.E.R.; ALBUQUERQUE, M.A.; CHARMELO L.L.; CAMPOS, J.C.F. \& SIMAS, F.B. Elementos da paisagem e a gestão da qualidade ambiental. Inf. Agropec., 21:20-44, 2000.

SCHAEFER, C.E.R.; KER, J.C.; GILKES, R.J.; CAMPOS, J.C.; COSTA, L.M. \& SAADI, A. Pedogenesis on the uplands of the Diamantina Plateau, Minas Gerais, Brazil: A chemical and micropedological study. Geoderma, 107:243-269, 2002. 
SPLECHTNA, B.E. \& KLINKA, K. Quantitative characterization of nutrient regimes of high-elevation forest soils in the Southern coastal region of British Columbia, Canada. Geoderma, 102:153-174, 2001.
UNITED STATES GEOLOGICAL SURVEY - USGS. Shuttle Radar Topography Mission (SRTM). 2005. Disponível em: <http://edc.usgs.gov/products/elevation/srtm>. Acesso em: nov. 2005.

R. Bras. Ci. Solo, 32:2081-2090, 2008 